\title{
Dimensiones políticas de la comunicación cinematográfica en la infancia y adolescencia
}

Pablo Salinas

Shawnee State University

\section{María Soledad Paz-Mackay y Omar Rodríguez. Politics of Children in Latin American Cinema. Lexington Books, 2019.}

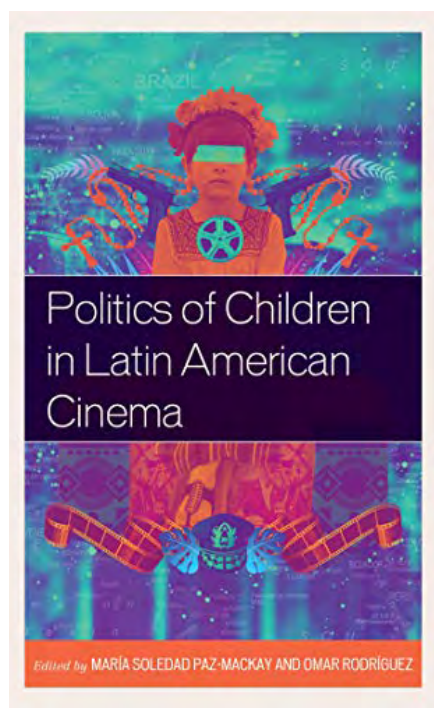

Politics of Children in Latin American Cinema es un volumen colectivo, con un eje más temático que genérico o teórico, interesado en la dimensión política de la construcción del infante latinoamericano a través del cine. Los doce capítulos tienen en común la intención de una puesta al día del proceso de subjetivación de la niñez con atención y reconocimiento a los elementos de la comunicación cinematográfica, especialmente al llamado "adult gaze". Me refiero en este caso a producciones adultas sobre un sujeto presentado como un recipiente vacío (el niño), capaz de ser llenado con elementos simbólicos que contribuyan a relaciones alegóricas infante-Estados nacionales.

Al momento de delimitar el sujeto de estudio, los editores reconocen el carácter amorfo que varía de acuerdo a los diversos campos de conocimiento que los doce autores de los capítulos abarcan. La niñez es entendida en algunos capítulos en un sentido más estricto, estrictamente hasta el inicio de la pubertad, mientras otros se enfocan más hacia el final de la adolescencia, con personajes participando en marchas políticas, ingresando en el servicio militar, entre otros aspectos. El hecho de ser una compilación en inglés que analiza producciones realizadas en español y portugués, con participación secundaria de diversas lenguas nativas regionales, nos obliga a referenciar temas relacionados con la traducción como el uso del término children, cuya doble acepción en inglés se relaciona directamente con la constante alusión alegórica de la paternidad en la relación Estado-individuo.

El libro consta de tres partes con cuatro capítulos en cada parte. Los doce textos se agrupan en subtemas bien escogidos, pero en nada excluyentes. En la articulación de estos se pueden notar superposiciones que no influyen en la calidad de la elección, puesto que entregan información de la extensa variedad de acercamientos y preocupaciones formales que abarcan el documental, la etnoficción, road film, la picaresca, el film animado, entre otros géneros. Esta diversidad, entendida también como caudal informativo, que a menudo se presenta dentro de las tres partes de la obra con transiciones inesperadas, nos invita a realizar a una reseña pormenorizada por cada capítulo. 
La primera parte, titulada "Representing Identity Struggles" inevitablemente trae a la memoria personajes como el Ojitos en Los Olvidados. Evidentemente, la compilación pudo precisar en la portada que los ensayos analizan películas producidas en el siglo XXI, excluyendo la ya aprovechada veta del melodrama mexicano (en sus diversas variantes) y su relación con el personaje infantil. Dentro de esta parte, en "Cinema and the Picaresque: The Art of Survival in the Paraguayan Film 7 cajas" se estudian las representaciones de las luchas de identidad teniendo como punto de arranque el alcance contemporáneo del género picaresco aplicado al cine. Aunque no estoy de acuerdo en considerar dentro del mismo género (o sub-género) a producciones tan disímiles como Pizza, birra, faso y las comedias de Cantinflas, entiendo, no obstante, que extender el concepto de lo picaresco abre una veta de lectura crítica muy fértil al relacionarla con producciones que dan una voz, por lo menos dentro de la diégesis, al personaje infantil en el cine. El segundo texto, "Familial Bonds and Developing Subjectivities in Veronica Reidel's Cápsulas", se enfoca más en las reformulaciones de las identidades familiares a través de traumas de la memoria. Teniendo en cuenta que el artículo trabaja eficientemente el concepto de "tristeza" como instrumental en la reelaboración de las relaciones afectivas familiares entre los protagonistas. Cabe señalar que "tristeza" fue acuñado por Frank Smith en un estudio de campo en las denominadas aldeas de origen maya, con atención a las fronteras socioculturales. Los protagonistas de Cápsulas son en cambio creaciones narrativas de una institución "letrada" y urbana anclada en la tradición del cine guatemalteco. Si el segundo texto se enfoca en la reconstitución familiar, "Michel's Franco Después de Lucía (2012): A Portrait of a Bullied Adolescent" identifica la brutal descomposición de lazos de fraternidad, de amistad y familiares. El uso del concepto de bullying como un tropo resulta útil para comprender la dimensión simbólica de la violencia a nivel nacional. La película es presentada como una alegoría de la erosión institucional mexicana donde no hay espacio ni tiempo para el recuerdo de la madre muerta. Si el concepto de la representación del infante en América Latina está íntimamente ligada a nuestra memoria cinematográfica con personajes icónicos como el Jaibo, Chachita, Pixote, etc., “A Uruguayan Girl: Anina (2013) by Alejandro Soderguit" rompe con esas expectativas al poner de relieve un film animado. El análisis en este capítulo es más formal que en los anteriores, especialmente en el tratamiento de la primera construcción de sentido, lo cual nos introduce a la película desde la perspectiva de la protagonista.

La segunda parte titulada "Constructing the Child Subjectivity" reafirma el alejamiento de la clasificación taxónomica del infante por edades y presenta a su vez temáticas de construcción de identidad en una perspectiva más relacionada con el entorno social. "Melancholia and Relajo in Gueros (2004)" subraya el ambiente melancólico y nostálgico explorado a través de la música, encontrando en ese empeño los momentos en que el concepto de "relajo" se torna central en la trama. La aplicación de este término como herramienta de análisis inevitablemente abre una relación interdiscursiva con lo carnavalesco o inclusive con acercamientos a la picaresca, tratada en uno de los capítulos precedentes. Llama la atención la reactualización de melancholia y especialmente de "relajo", este último anclado inicialmente en los años sesenta, como elementos centrales para 
comprender las dinámicas del sujeto y la subjetivación en una película de 2015. Por otro lado el segundo capítulo, "Re-mediating Adolescent Subjetivities: Indigeneity and the Gendered 'Dividual' in El niño pez (2009) and Feriado (2014)" es el primero que analiza películas de dos países diferentes, Argentina y Ecuador, teniendo como hilo conductor el común interés en la representación étnica y de género. Este acercamiento, alternativo a otros conceptos de otredad más anclados en estéticas neorrealistas, resulta apropiado para enlazar dos producciones de tradiciones y peso cinematográfico distinto. El tercer capítulo "When Children Direct a Film, What Do They Talk About" es tan ambicioso como su título al analizar cuatro películas consideradas marginales, en las que se encuentra un documental. El estudio de estas producciones realizadas por adolescentes se incrusta directamente en la veta autoreflexiva que anuncia la introducción de esta recopilación. No obstante, la preocupación centrada en el extenso proceso de comunicación cinematográfico, con especial enfoque en la enunciación, acentúa un salto abrupto entre los textos de esta segunda parte, brecha tal vez evitada si este apartado se hubiera articulado con la introducción. El cuarto capítulo "Under the Wing of the Law, Juizo (2008) by Maria Augusta Ramos" es el único que analiza una producción en portugués, escogiendo en este esfuerzo un híbrido entre el documental y la ficción. Aquí parece presentarse un debate intertextual al echarse por tierra inicialmente el dualismo postcolonial primer / tercer mundo, mientras que, al explorar, las posiblidades de la mise en scene, proceder a denunciar la herencia etnocultural colonial, todavía presente en el sistema judicial brasileño.

La tercera parte Revisiting the Traumatic Past se enfoca de lleno en el tema de la memoria en tiempos postrevolucionarios y postdictatoriales. El primer capítulo "On an Off the Road with the Children of Che Guevara: Viva Cuba and Infancia clandestina (2011)" engarza las películas que focalizan al infante con la estética del road movie. Además de los directos paralelos con Diarios de motocicleta, este capítulo contrapone la estética de estas dos producciones a los acercamientos estéticos del Tercer Cine en la representación de la infancia. A diferencia del capítulo anterior, "Transmission of Memory in Héctor Gálvez's Paraíso" se enfoca en protagonistas que bordean los últimos años de la adolescencia. La utilización de conceptos como el de memoria o postmemoria resulta acertada al ser Paraíso una película que orgánicamente se nutre de la experiencia migrante y donde son evidentes los vasos comunicantes entre ficción y realidad o por lo menos una estética e intención documental. El texto inscribe el concepto de "incipient postmemory" que resultaría también útil al aplicar preguntas referidas a quién recuerda cuando se habla de memoria en el cine latinoamericano. Por otro lado, "What's Wrong with My Name? Appropriation, Identity, and Familial Ties in Cautiva (2005)" al centrarse en la revisión de un pasado traumático, inevitablemente inserta el discurso en la representación de las luchas sobre y por la identidad, preocupación abordada en la primera parte de esta recopilación. El uso del concepto de "apropiación" resulta doblemente productivo, no solamente en la evocación al pasado traumático, sino en lo que el texto denomina transferencia de saberes a las nuevas generaciones. En este sentido el capítulo avanza en paralelo entre la historia de la adolescente Cristina / Sofía y el constante hincapié en su contexto extradiegético. 
El último capítulo, "Children, Broken Families, and National Trauma in Contemporary Dictatorship Films in Chile: Andres Wood's Machuca (2004) and Pablo Larraín's No (2012)” pone en cuestión la aplicabilidad de conceptos como el de "postmemoria", pensados para casos específicos, en este caso el Holocausto. Este análisis tiene el mérito de relacionar conflictivamente las narrativas de divorcio en las dos películas analizadas, con conceptos clásicos en el análisis literario como el de los romances en las ficciones fundacionales de Doris Sommer.

Politics of Children in Latin America es una obra de consulta referencial que se ofrece como necesario complemento a otros estudios constantemente citados en esta recopilación. Me refiero a Representing History, Class, and Gender in Spain and Latina America: Children and Adolescents in Film, Screening Minors in Latin American Cinema o, más a la distancia, los trabajos de Marianne Hirsch sobre narrativa y postmemoria. La más notoria contribución de esta recopilación es entrelazar los debates de la memoria con la representación de protagonistas menores de edad en textos que muchas veces van a contracorriente con la tradición cinematográfica latinoamericana. En lugar de naturalizar la enunciación, los autores subrayan la artificialidad de la perspectiva adulta sobre los protagonistas menores de edad, primer paso obligado para enfocarse en aspectos sociopolíticos de los filmes abordados en los capítulos. Aunque no todas las entradas atacan la perspectiva política con la misma intensidad, esta recopilación amplía con creces trabajos interesados en la dimensión política de la comunicación cinematográfica con un enfoque temático centrado en protagonistas niños y adolescentes. Espero que estos mismos acercamientos y propuestas circulen ya, seguramente de forma más individual, en los idiomas en los que se gestaron las películas que originaron los análisis. 